\title{
Comment on: Safety and feasibility of hemodynamic pulmonary artery pressure monitoring using the CardioMEMS device in LVAD management
}

\author{
$\mathrm{J} \mathrm{Breda}^{1}$ \\ ${ }^{1}$ Miami Transplant Institute
}

May 18, 2021

\begin{abstract}
The called burden of cardiac heart failure ( $\mathrm{CHF})$ on healthcare systems and economies remains large and a major factor contributing to this burden is the high hospital admission rate for acute decompensated heart failure. These repeated heart failure hospitalizations ( $\mathrm{HFH}$ ) not only exert a high burden on healthcare systems, but also impact patient quality of life and have been associated with impaired prognosis and reduced life expectancy. The need for remote monitoring has become extremely important, mainly based on devices capable of measuring intracardiac filling pressures. If we assume that hemodynamic congestion precedes clinical congestion, the hemodynamic monitoring could be able to detect early signs of congestion and enables clinicians to intervene in a pre-symptomatic phase avoiding hospital admission. Dr. Veenis JF and colleagues present the results of implanting the CardioMEMS device in 10 patients who underwent heartmate 3 implantation. The authors describe the study design based on an earlier publication by the same author. The authors argue that the use of this device will allow the monitoring of patients pre, during hospitalization and after implantation, with a possible reduction in the number of readmissions for allowing the diagnosis and treatment of complications related to ventricular failure and volume overload.
\end{abstract}

Comment on: Safety and feasibility of hemodynamic pulmonary artery pressure monitoring using the CardioMEMS device in LVAD management

The called burden of cardiac heart failure ( $\mathrm{CHF}$ ) on healthcare systems and economies remains large and a major factor contributing to this burden is the high hospital admission rate for acute decompensated heart failure. These repeated heart failure hospitalizations (HFH) not only exert a high burden on healthcare systems, but also impact patient quality of life and have been associated with impaired prognosis and reduced life expectancy. The need for remote monitoring has become extremely important, mainly based on devices capable of measuring intracardiac filling pressures. If we assume that hemodynamic congestion precedes clinical congestion, the hemodynamic monitoring could be able to detect early signs of congestion and enables clinicians to intervene in a pre-symptomatic phase avoiding hospital admission.

Dr. Veenis JF and colleagues present the results of implanting the CardioMEMS device in 10 patients who underwent heartmate 3 implantation. The authors describe the study design based on an earlier publication by the same author. The authors argue that the use of this device will allow the monitoring of patients pre, during hospitalization and after implantation, with a possible reduction in the number of readmissions for allowing the diagnosis and treatment of complications related to ventricular failure and volume overload.

The use of CardioMEMS was first addressed in the 2011 US CHAMPION trial (CardioMEMS Heart Sensor Allows Monitoring of Pressure to Improve Outcomes in NYHA Class III Heart Failure Patients). This singleblind randomized controlled trial enrolled 550 patients in NYHA class III with a previous HF hospitalization. The primary efficacy outcome was the rate of hospital admissions between the treatment and control group. 
The HF-related hospitalization rate was significantly lower in the intervention group as compared to the control group during the complete randomized period, with a mean follow-up time of 15 months (hazard ratio (HR) $0.63,95 \%$ confidence interval (CI) 0.52-0.77). (1)

We agree that the pulmonary artery pressure (PAP) guided adjustment of medications would likely result in a reduction in HF readmissions and the authors added the possibility of better preoperative management and during ICU stay for these patients after Heartmate 3 implantation. Although, there are some scenarios where this monitoring might be suboptimal. The most ideal parameter would be the pulmonary capillary wedge pressure or left atrial pressure, for example, in patients with pulmonary hypertension and primary pulmonary disease or in the case of increased pulmonary vascular resistance. (2)

Despite these factors the device analyzed by the authors in the present article still remains very promising in the near future. More than reducing readmissions, the use of this device in the preoperative period and during hospitalization after surgery could be useful in the management of these patients in critical periods. Finally, we can still remember the great importance of ways of remote monitoring of patients, especially at times like the one we faced during the Covid-19 pandemic. (3)

References

1. Abraham WT, Adamson PB, Bourge RC, Aaron MF, Costanzo MR, Stevenson LW, Strickland W, Neelagaru S, Raval N, Krueger S, Weiner S, Shavelle D, Jeffries B, Yadav JS Wireless pulmonary artery haemodynamic monitoring in chronic heart failure: a randomised controlled trial., CHAMPION Trial Study Group.Lancet. 2011 Feb 19; 377(9766):658-66.

2. Maurer MS, Adamson PB, Costanzo MR, Eigler N, Gilbert J, Gold MR, Klapholz M, Saxon LA, Singh JP, Troughton R, Abraham WT Rationale and Design of the Left Atrial Pressure Monitoring to Optimize Heart Failure Therapy Study (LAPTOP-HF). J Card Fail. 2015 Jun; 21(6):479-88.

3. Radhoe SP, Veenis JF, Brugts JJ Invasive Devices and Sensors for Remote Care of Heart Failure Patients. Sensors 2021, 21(6), 2014. 